AperTO - Archivio Istituzionale Open Access dell'Università di Torino

\title{
The JEM-EUSO instrument
}

\section{This is the author's manuscript}

Original Citation:

Availability:

This version is available http://hdl.handle.net/2318/1653122

since 2017-11-25T12:17:37Z

Published version:

DOI:10.1007/s10686-014-9418-x

Terms of use:

Open Access

Anyone can freely access the full text of works made available as "Open Access". Works made available under a Creative Commons license can be used according to the terms and conditions of said license. Use of all other works requires consent of the right holder (author or publisher) if not exempted from copyright protection by the applicable law. 


\title{
The JEM-EUSO instrument
}

\author{
The JEM-EUSO Collaboration
}

Received: date / Accepted: date

\begin{abstract}
In this paper we describe the main characteristics of the JEMEUSO instrument. The Extreme Universe Space Observatory on the Japanese Experiment Module (JEM-EUSO) of the International Space Station (ISS) will observe Ultra High-Energy Cosmic Rays (UHECR) from space. It will detect UV-light of Extensive Air Showers (EAS) produced by UHECRs traversing the Earth's atmosphere. For each event, the detector will determine the energy, arrival direction and the type of the primary particle. The advantage of a space-borne detector resides in the large field of view, using a target volume of about $10^{12}$ tons of atmosphere, far greater than what is achievable from ground. Another advantage is a nearly uniform sampling of the whole celestial sphere. The corresponding increase in statistics will help to clarify the origin and sources of UHECRs and characterize the environment traversed during their production and propagation. JEM-EUSO is a 1.1 ton refractor telescope using an optics of $2.5 \mathrm{~m}$ diameter Fresnel lenses to focus the UV-light from EAS on a focal surface composed of about 5000 multianode photomultipliers, for a total of $\simeq 3 \cdot 10^{5}$ channels. A multi-layer parallel architecture handles front-end acquisition, selecting and storing valid triggers. Each processing level filters the events with increasingly complex algorithms using FPGAs and DSPs
\end{abstract}

The JEM-EUSO Collaboration

The full author list and affiliations are given at the end of paper; corresponding authors:

Marco Casolino

RIKEN, 2-1 Hirosawa, Wako-shi, Japan

INFN \& Univ. Rome Tor Vergata, Rome, Italy

E-mail: casolino@roma2.infn.it

Fumiyoshi Kajino

Konan University, Faculty of Science and Engineering, Department of Physics

E-mail: kajino@konan-u.ac.jp

Lech Wiktor Piotrowski

RIKEN, 2-1 Hirosawa, Wako-shi, Japan

E-mail: lech.piotrowski@riken.jp 
Table 1 Parameters of the mission

\begin{tabular}{|l|l|}
\hline Mass & $1153 \mathrm{~kg}$ \\
\hline Dimensions & $290 \mathrm{~cm}(\mathrm{x}) \times 270 \mathrm{~cm}(\mathrm{y}) \times 160 \mathrm{~cm}(\mathrm{z})$ \\
\hline Entrance Pupil Diameter (EPD) & $2300 \mathrm{~mm}$ \\
\hline Lens diameter & $2650 \mathrm{~mm}$ \\
\hline Field of view (FoV) & $\pm 30^{\circ}$ \\
\hline F-number & 1 \\
\hline Spot size & $<2.8 \mathrm{~mm}$ \\
\hline Power consumption & $516.7 \mathrm{~W}$ \\
\hline Data output & $\begin{array}{l}263 \mathrm{~KB} / \mathrm{s}+\text { Lidar, HK, AM } \\
\text { addit. } 10 \mathrm{~GB} / \text { day stored }\end{array}$ \\
\hline
\end{tabular}

to reject spurious events and reduce the data rate to a value compatible with downlink constraints.

Keywords UHECR · International Space Station · cosmic rays

\section{Introduction}

JEM-EUSO is a Fresnel-optics refractive telescope devoted to the observation of Ultra High Energy Cosmic Rays (UHECR) $[1,2]$ through the detection of ultra-violet (UV) fluorescence light emitted by particles producing showers in the atmosphere (see fig. 1). Its goal is to investigate the nature of UHECR, determining the origin, and identifying their sources and measuring the different spectra. This will be achieved by $\mathrm{a} \simeq 10$-fold increase in statistics obtained from the large area under observation, with respect to the existing on-ground experiments. Further goals include Earth observation in the UV-band and the study of atmospheric phenomena including Transient Luminous Events [3] and meteors [4]. Fundamental particle physics aims include the search for nuclearites [4], search for high energy neutrinos [5], heavy dark matter candidates. The scientific goals of JEM-EUSO are described in detail in [6-8].

The telescope will orbit the Earth every $\simeq 90$ minutes on board the International Space Station (ISS) at an altitude of $330-400 \mathrm{~km}$, making observations in the dark part of the Earth, about 40 minutes per orbit. The main parameters of the mission are shown in tab. 1.

\section{Principle of observation}

JEM-EUSO will be measuring the UV (290-430 nm) light coming from EAS induced by UHECR interaction in the Earth's night atmosphere. A schematic view of the telescope is shown in fig. 2. The UV light is emitted by fluorescence and Cherenkov radiation in the atmosphere. The UV light from the shower is focused through a wide field of view $\left( \pm 30^{\circ}\right)$ diffractive optics employing Fresnel lenses. The light is detected by $\mathrm{a} \simeq 3 \cdot 10^{5}$ pixel focal plane electronics which records the track of the EAS with a time resolution of $2.5 \mu \mathrm{s}$ and an 


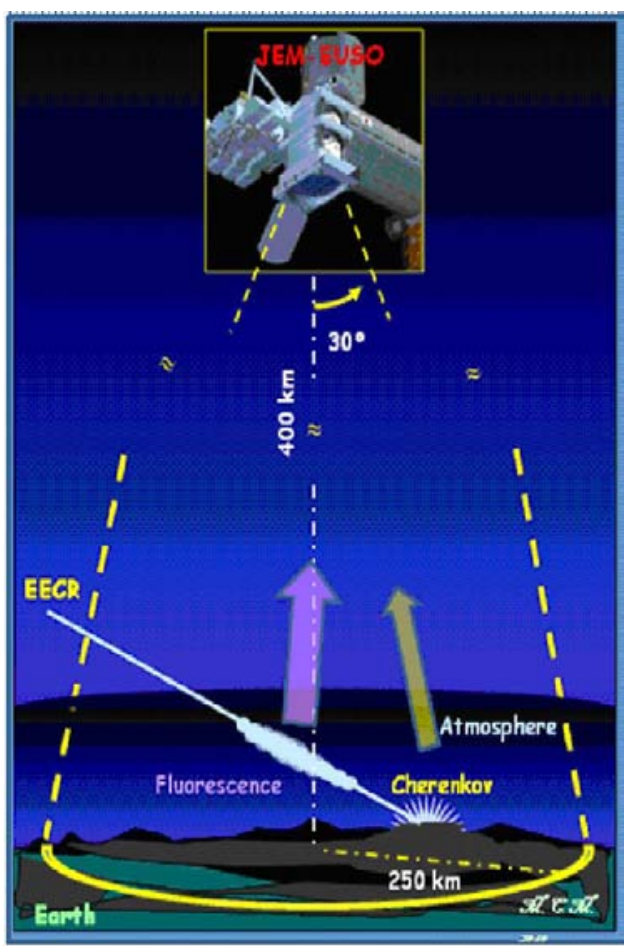

Fig. 1 Artistic view of the JEM-EUSO principle of observation. The telescope detects from the ISS both fluorescence and Cherenkov light, produced by the EAS of UHECR propagating through the atmosphere.

angular resolution of $0.074^{\circ}$, corresponding to a pixel size of about $0.51 \mathrm{~km}$ (0.61 km at the edge of FoV) at $400 \mathrm{~km}[9]$. The spatial and temporal profile of the event will allow to determine the energy and direction of the primary particles (with an accuracy better than several degrees (fig. 3(a)). fig. 3(b) shows a simulation of the time profile of UV photons (of fluorescence and Cherenkov origin) as observed from JEM-EUSO. A particle of $E \simeq 10^{20} \mathrm{eV}$ penetrating the Earth's atmosphere has an interaction length of $40 \mathrm{~g} / \mathrm{cm}^{2}$. The number of generated secondary particles $\left(\simeq 10^{11}\right)$ is proportional to the shower energy and is largely dominated by electrons and positrons. The total energy carried by the charged secondary particles is converted into fluorescence photons through the excitation of the air nitrogen molecules. The fluorescence light is isotropic and proportional to the number of charged particles in the EAS. It is however dependent on the pressure (altitude) and slightly on the temperature and humidity.

The telescope can point nadir, observing a circular area of about $380 \mathrm{~km}$ in diameter, with a instantaneous geometrical area greater than $1.4 \cdot 10^{5} \mathrm{~km}^{2}$. It can be tilted to $30^{\circ}$. This inclined configuration increases the field of view 


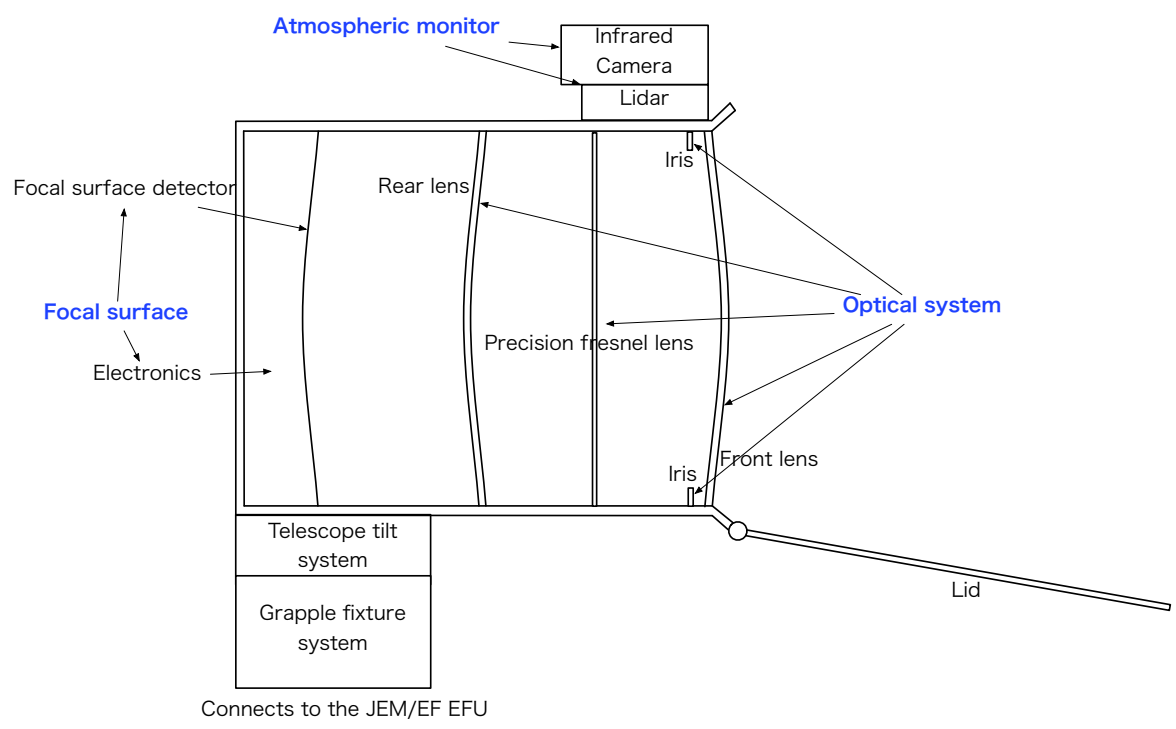

Fig. 2 Conceptual design of the JEM-EUSO system: three Fresnel lenses focus the light on the focal surface.

but also the threshold energy to detect primary particles, so it is suited to high statistic studies of the high energy part of the spectrum.

\section{Optics}

The main requirements for JEM-EUSO optics are:

- A large optical system to increase the number of photons collected and thus lower the threshold for minimum energy detection of UHECR.

- A large Field-of-View (FoV) to increase the number of events observed.

This results in a wide-field optics, with a low focal number (the ratio between the optics aperture and the focal length) $F / 1$. The use of Fresnel lenses realized in Poly(Methyl MethAcrylate) - PMMA - allows to build a refractor telescope capable of meeting these requirements with the constraints posed by spaceborne experiments. Furthermore, the reduced thickness of the lenses allows to reduce the mass of the optics resulting in a light system capable of withstanding launch vibration and thermal expansion.

The conceptual design of the JEM-EUSO optics is shown in fig. 2. UV photons coming from the atmospheric showers are focused on the pixels of the optical focal surface.

Status and Technology Readiness Level (TRL) is $5^{1}$. Three complete models based on the JEM-EUSO concept have been realized in RIKEN. A $1.5 \mathrm{~m}$

1 TRL 5 corresponds to component and/or breadboard validation in relevant environment. 


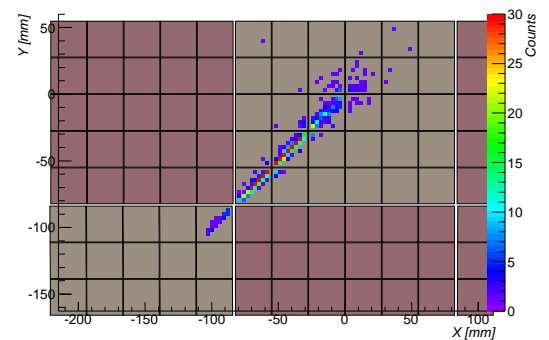

(a) Spatial profile

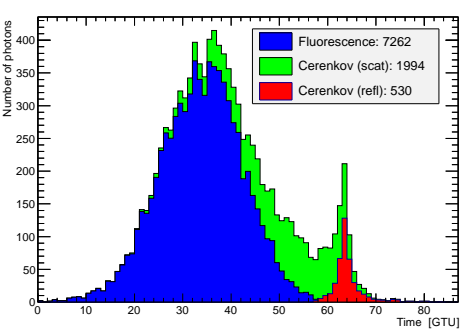

(b) Time profile

Fig. 3 Left: Spatial profile of photons from an EAS caused by a $10^{20} \mathrm{eV}$ proton with zenith angle $60^{\circ}$. Each square represents a Multi Anode Photomultipler (MAPMT) with 64 channels, arranged in units of $6 \times 6$ phototubes. Subsections of 6 different PhotoDetector Modules (PDMs) are shown. The small squares show the number of photons detected by each channel of the MAPMT. This event is crossing diagonally three PDMs, with the shower starting to develop in the bottom left PDM and continuing in the right top PDM. Right: Time profile of photons, obtained summing all photons of the previous picture detected on each Gate Time Unit (GTU) $(2.5 \mu \mathrm{s})$. It is possible to see the contribution to the signal from the three components (UV light -blue, Cherenkov peak - red, scattered Cherenkov green).

circular lens system (fig. 4), the central part of the optics, has been tested in Marshall Space Flight Center (MSFC) and performances have exceeded the requirements, A two $1 \times 1 \mathrm{~m}$ square lens telescope has been installed in Telescope Array site in Utah. A three $1 \times 1 \mathrm{~m}$ square lens telescope has been mounted in the gondola for a CNES-sponsored campaign of balloon flights.

The diffractive surface of the $2^{\text {nd }}$ lens has grooves of height $0.694 \mathrm{~mm}$ and width varying from $6 \mu \mathrm{m}$ to $100 \mu \mathrm{m}$. The manufacturing tolerance for the groove height is $\pm 10 \%$, or an accuracy better than $\pm 0.069 \mathrm{~mm}$.

The optical system is divided into (fig. 5):

- $1^{\text {st }}$ (nadir) lens (curved doublet Fresnel lens): the lens facing space. The first lens is coated with Cytop [10] to protect it from the atomic oxygen present at the orbit of the ISS.

- Iris, needed to reduce the stray light and the entrance pupil diameter on axis 


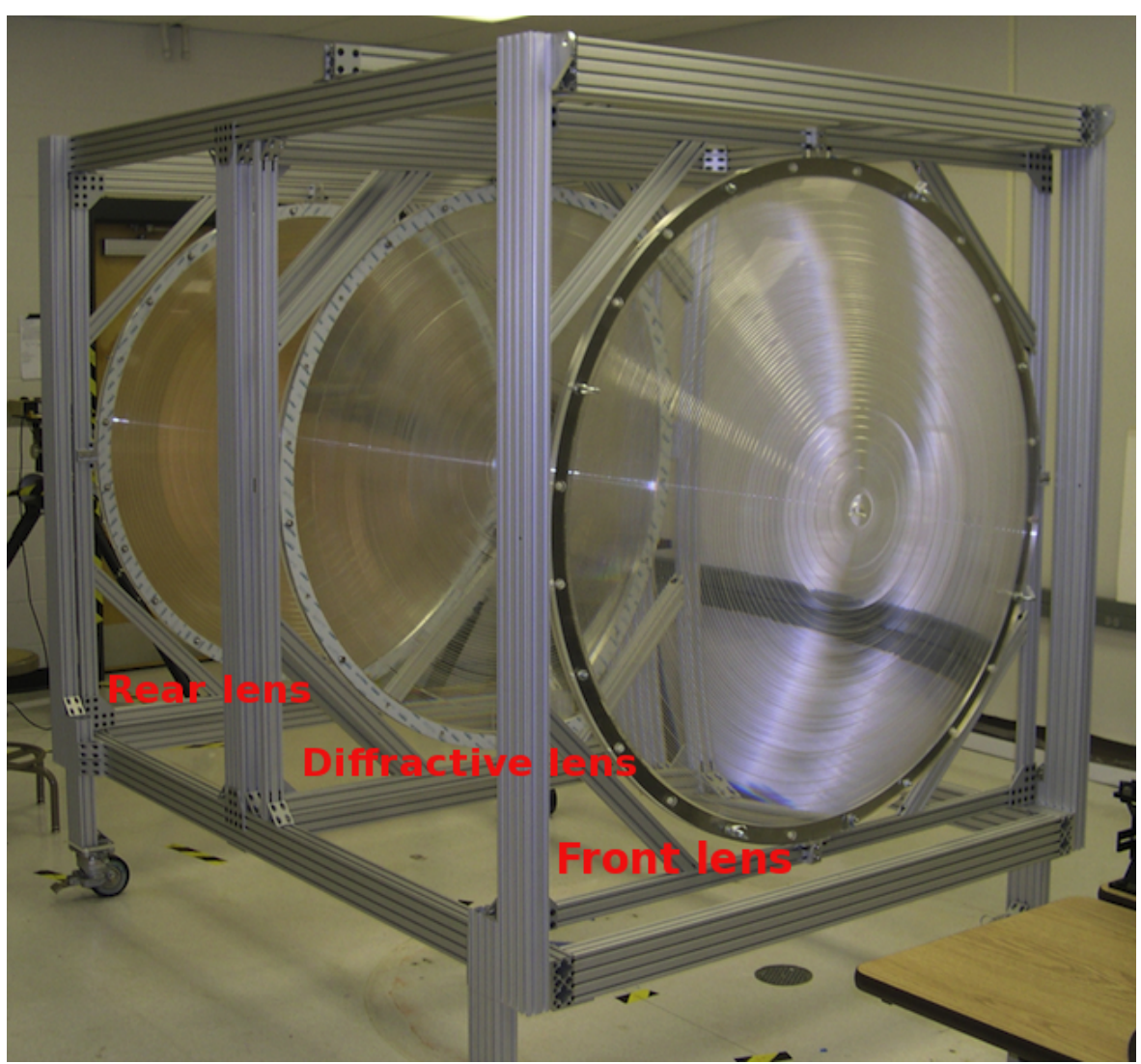

Fig. 4 The $1.5 \mathrm{~m}$ diameter three lens optics under test at MSFC.

$-2^{\text {nd }}$ lens (Fresnel + diffractive surface lens): serves as field lens to reduce the vignetting factor and as a chromatic corrector. The two surfaces of the $2^{\text {nd }}$ lens in the JEM-EUSO optics are characterized by:

- Fresnel surface, acting as a "field lens". This surface is able to reduce the spot size, improving the RMS spot size from $5 \mathrm{~mm}$ to $2.5 \mathrm{~mm}$.

- Diffractive surface to reduce the wavelength aberrations. Emission of UV photons by the nitrogen molecules of the atmosphere ranges from 290 to $420 \mathrm{~nm}$, resulting in chromatic aberration due to the variation of the PMMA refractive index. To compensate for this effect we use of a diffraction grating, which has opposite colour dispersion.

$-3^{r d}$ lens (curved doublet Fresnel lens): focuses light on the focal surface

- Other parts of the optical system include the UV filter glued to the photomultipliers, the lens frame and focus-adjust-system. 


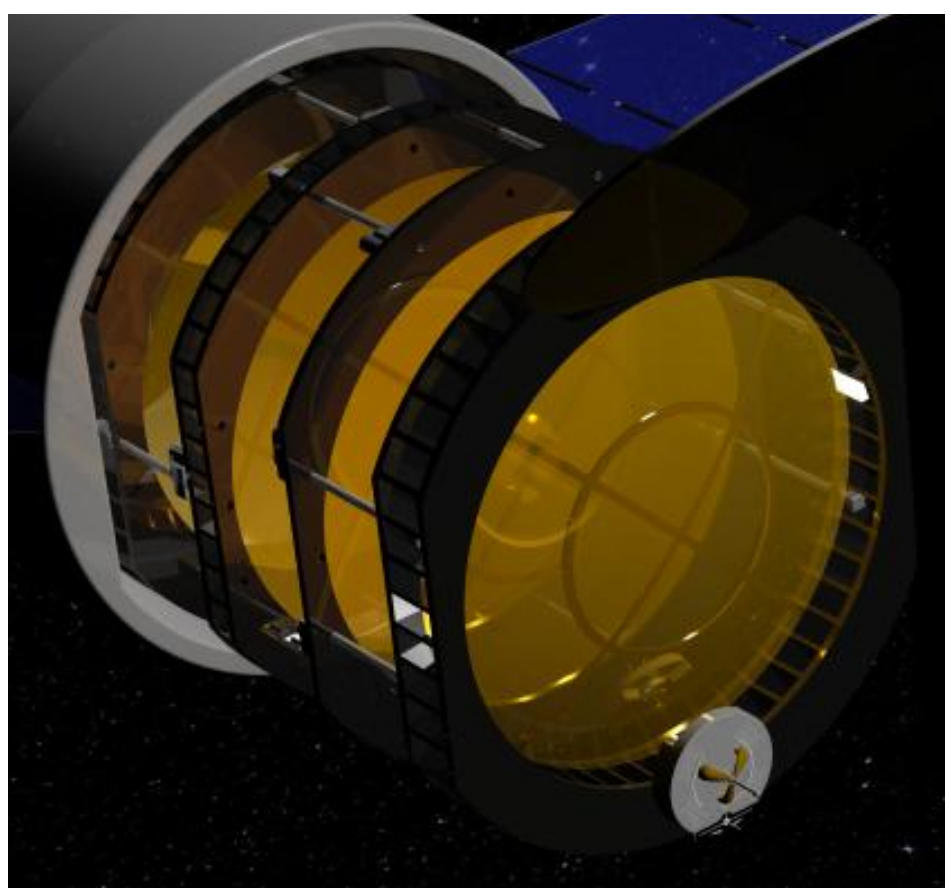

Fig. $5 \mathrm{CAD}$ model of the JEM-EUSO structure and its main elements, accommodated in the Dragon capsule, after unfolding. The detector will be attached to the ISS through the focal surface. The front lens (bottom-right of the picture) looks toward the Earth.

\section{Focal surface and detector electronics}

The Focal Surface (FS) of JEM-EUSO, located in the focal spot of the optics, has a spherically curved surface of about $2.5 \mathrm{~m}$ in diameter and is covered with about 5,000 Multi-Anode PhotoMultiplier Tubes (MAPMTs, Hamamatsu R11265-M64), each with 64 pixels. The FS consists of 137 Photo-Detector Modules (PDMs) (see fig. 6). Each PDM comprises a $3 \times 3$ set of Elementary Cells (ECs). Each EC is formed by a $2 \times 2$ array of MAPMTs (see fig. 7 ). The total number of pixels is 315648, with a spatial resolution of $0.074^{\circ}$ for each pixel. The FS detector converts photons into electrical pulses with 2 ns width, which are accumulated by the electronics during a Gate Time Unit (GTU) of $2.5 \mu \mathrm{s}$.

\subsection{Data acquisition and reduction}

The data acquisition system is designed to maximize the observation capabilities of JEM-EUSO, monitor system status, autonomously take all actions to maintain optimal acquisition capabilities and handle off-nominal situations. CPU and electronics are based on hardware successfully employed in space ex- 


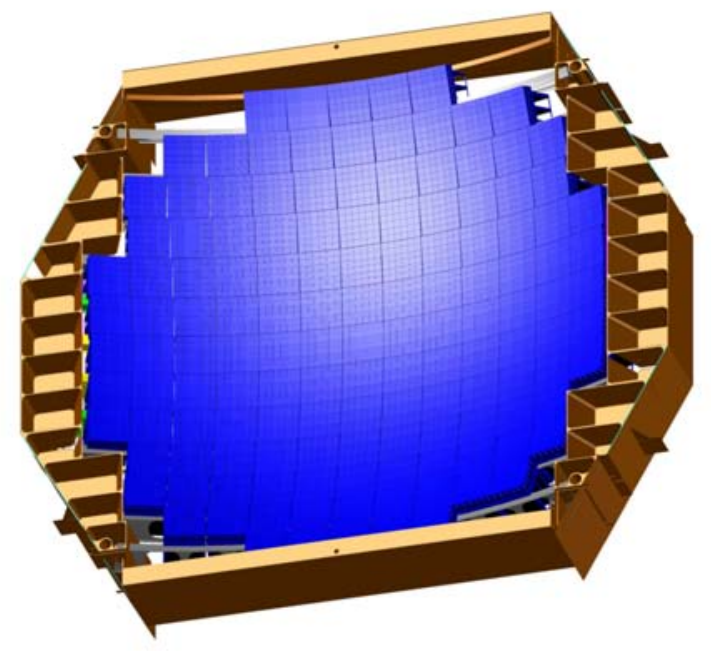

Fig. 6 Arrangement of the $137 \mathrm{PDMs}$ on a $\mathrm{R}=2505 \mathrm{~mm}$ spherical surface - the Focal Surface.

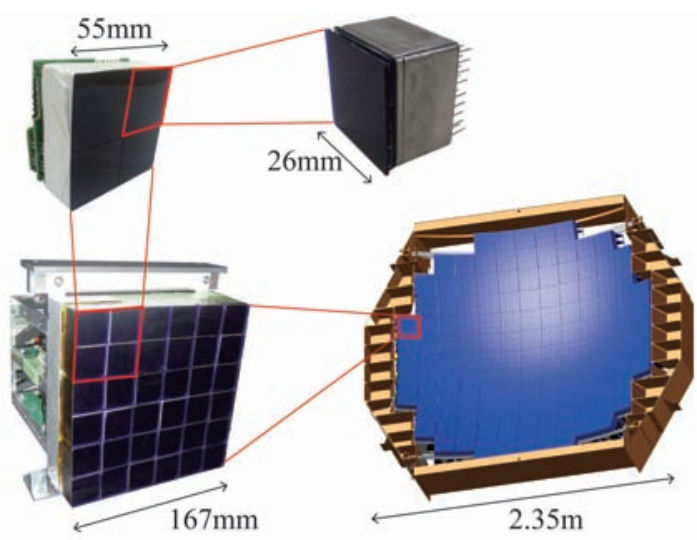

Fig. 7 Structure of the Focal Surface. The $2.5 \mathrm{~m}$ surface is divided in 137 PDM modules. Each PDM contains 36 Multi-Anode Photomultipliers (Hamamatsu Ultra-Bialkali R1126564 ), each with 64 independent channels. The bottom left corner shows the prototype of the mechanical structure with 36 PMTs installed.

periments such as PAMELA, Altea, Agile etc., taking into account recent developments in microprocessors and FPGA technology. Acquisition techniques and algorithms are also derived from the technological development performed in these missions. Rad-hard technology will be employed, with ground beam 
tests at accelerator facilities such as GSI, HIMAC to qualify and test radiation resistance of new devices. Space qualified devices will be employed for mission-critical items such as the CPU.

The data handling scheme is based on a parallel architecture capable of reducing at each level the amount of data through a series of triggers controlling an increasingly growing area of the focal surface [11]. This is required to reduce the $\simeq 142 \mathrm{~GB} / \mathrm{s}$ output on the focal surface to the $\simeq 3 \mathrm{~GB} /$ day which can be downlinked on the ground. Each board receives the data and sends them to the higher level of processing if the trigger conditions are satisfied.

\subsection{PMTs}

The MAPMT unit (see fig. 8), which has been developed by RIKEN in collaboration with Hamamatsu Photonics, has an ultra-bialkali (quantum efficiency higher than 35\%) photo-cathode which converts photons into electrons. Each PMT has 64 pixels arranged in an array of $8 \times 8$ squares (pitch of $2.88 \mathrm{~mm}$ ) with a maximum sensitive area of $23.04 \mathrm{~mm}$ x $23.04 \mathrm{~mm}$. Each tube is equipped with $0.8 \mathrm{~mm}$ thick UV-transmitting filter in a shape of Winston cone to recover dead area at the border of PMT and between tubes. The device has a metal channel dynode structure with 12 stages, providing a gain of the order of $10^{6}$ at $0.9 \mathrm{kV}$ with a tapered voltage divider. Anode pulse rise-time is about $1 \mathrm{~ns}$ with transit time spread of about $0.3 \mathrm{~ns}$. Cross talk between pixels is below $1 \%$. Efficiency of the pixels (ratio of photoelectrons produced at anodes relative to photons hitting the cathode) is around $22 \%$ with a dispersion between pixels of $30 \%$. The distance between pixels, less than $0.1 \mathrm{~mm}$, is negligible.

\subsection{High Voltage}

The classical way to polarize a PMT is to use a voltage divider: in parallel with the dynodes, a resistor chain with the $\mathrm{HV}$ at one extremity and ground at the other develops voltages across the resistors, and these voltages are applied to the dynodes. This is simple, but very ineffective: in the case of JEM-EUSO, with dynamics of 100 (to be explained in the next paragraph on switches), the current between last dynode D12 and anodes can reach $2.5 \mathrm{~mA}$ per EC-unit (4 PMTs, with one common HV for them). So the current in the divider should be 100 times bigger, that is $250 \mathrm{~mA}$ per $\mathrm{HV}$ unit. Then at $900 \mathrm{~V}$ the power used is $1.25 \mathrm{~W} / \mathrm{HV}$. There are $1233 \mathrm{EC}$ units, hence $1233 \mathrm{HV}$ in the focal surface, which corresponds to a power dissipated of $2.8 \mathrm{~kW}$ - way too much.

In fig. 9 we show the adopted Cokroft-Walton (CW) circuit, a succession of voltage multipliers which is suited for this application, since all inter-dynodes voltages are equal to about $60 \mathrm{~V}$.

The total power of the full FS with gains of $10^{6}$ and a dynamical range of 100 with one CW per EC-unit is $50 \mathrm{~W}$ due to the low impedance of each output to the dynodes (current is drawn only when needed). The power drawn 

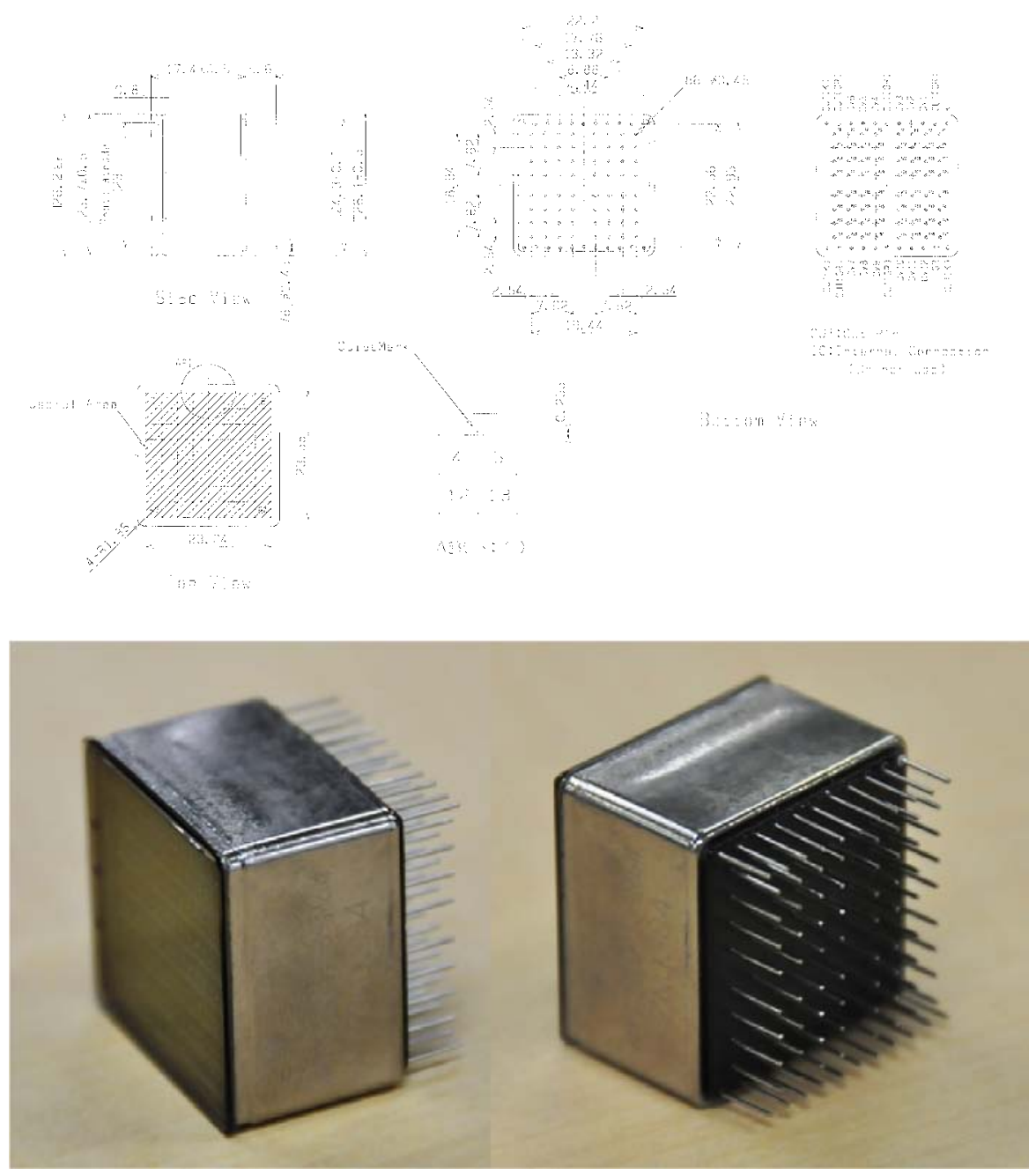

Fig. 8 Hamamatsu R11265-M64 Multianode Photomultiplier Tube unit.

is almost independent on the number of HVPS used. It depends only on the number of PMTs which is constant. The decision to have one CW per EC unit is a compromise between complexity (the total number of HVPS) and the risk of failure for a five year mission in space.

Protection of PMTs and measurements of intense light; switches

Most of the time, the light impinging on the pixels will be close to the background: $1.5 \frac{\mathrm{pe}}{\mathrm{GTU} \cdot \mathrm{pixel}}$. However, there are some intense lights which could damage the PMTs, like Transient Luminous Events (TLE), meteors, etc. For ex- 


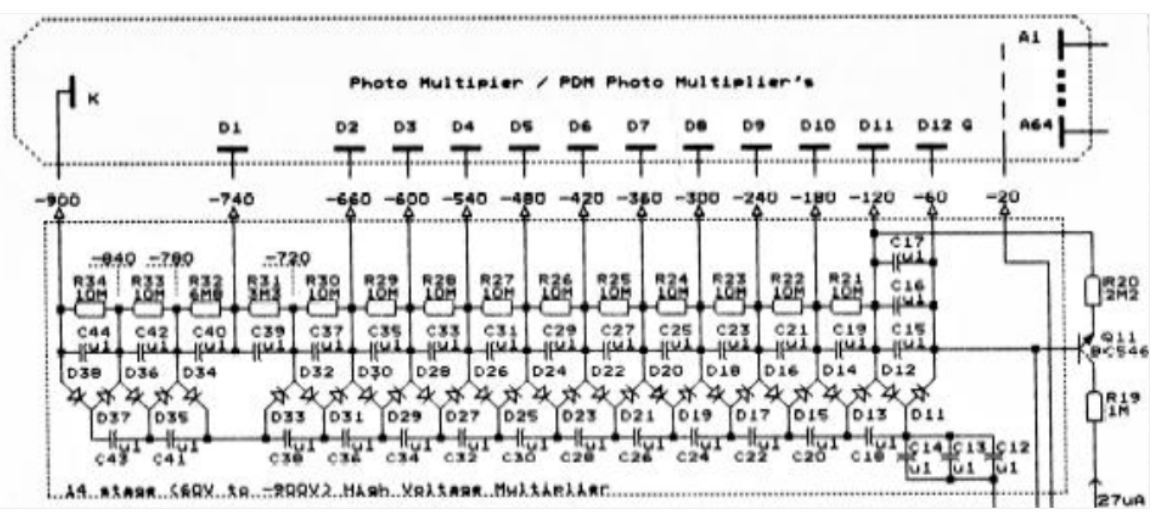

Fig. 9 Scheme of the CW circuit.

ample, TLEs can reach about $10^{6}$ times the background light intensity. The program of the mission includes the study of such lights, so the PMTs cannot be simply switched off. Therefore, it has been decided to include in each HVPS a system to reduce the current drawn by the PMT in case of strong illumination. The chosen solution is to switch down the voltage applied to the cathode, while keeping all other voltages applied to dynodes untouched. This means that only the collection efficiency is varied, not the gain which depends only on the voltages on the dynodes (the gain stays at $10^{6}$, hence the operation is always in single pe mode). The number of pe emitted by the photocathode follows the light intensity, but the number of pe reaching the first dynode is reduced. The command to switch down is given by measuring the D12 current in the HVPS and setting two thresholds: a high current

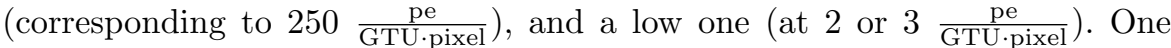
discriminator switches down by a factor of 100, the other up to go back to the original collection efficiency. A small logic chip inside the HVPS box controls the operations. It was found that if we apply to the photocathode the same voltage as to the first dynode, the efficiency is divided by 100 . If the voltage is set to $0 \mathrm{~V}$, the efficiency is reduced by another factor of 100 . With the cathode voltage at $900 \mathrm{~V}$, the PMT can measure from around 0 up to $150 \frac{\text { pe }}{\text { GTU.pixel }}$. At $740 \mathrm{~V}$, from about 100 to $15000 \frac{\text { pe }}{\text { GTU.pixel }}$, and finally at $0 \mathrm{~V}$, from 10000 to $1.5 \cdot 10^{6} \frac{\mathrm{pe}}{\text { GTU.pixel }}$. The switching is fast because the capacitance of the cathode is smaller than that of the full tube.

The galvanic insulation between command (logic pulse) and $\mathrm{HV}$ is realized through a transformer, which is faster than optical insulation. That way, whatever the light intensity, the phenomenon can be measured, and the tubes are protected. 


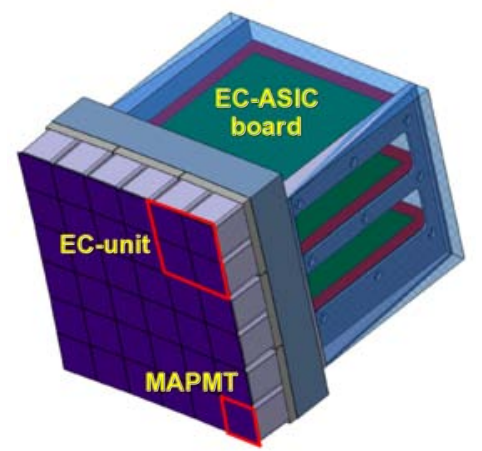

Fig. 10 Pictures of the assembled PDM unit, consisting of 36 MAPMTs, 9 EC-units, for a total of 2304 channels.

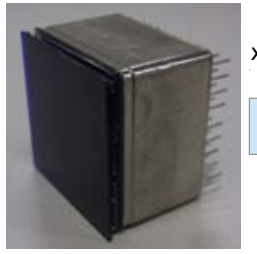

MAPMT module

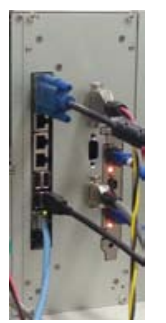

CPU

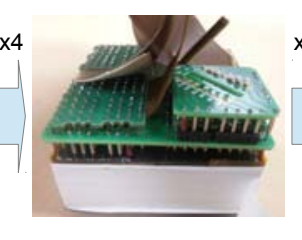

EC-unit (4 MAPMTs)

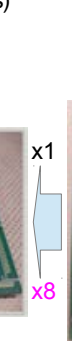

CCB

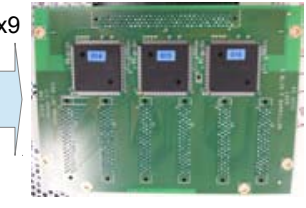

EC-ASIC board

$\mathrm{x} 6$

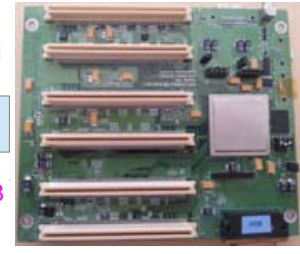

PDM board

Fig. 11 Scheme of the signal flow in the JEM-EUSO project. The subsystem multiplication numbers in black are for precursor EUSO-TA and EUSO-BALLOON, while pink are for the full JEM-EUSO mission.

\subsection{Focal Surface Electronics}

The 137 PDMs comprising the Focal Surface are each composed by 36 MAPMTs (fig. 10). They are individually read by an ASIC SPACIROC chip. Then data are sent to an FPGA that reads the whole PDM, named PDM-board (fig. 11).

The PDM-board stores the data coming from the 36 MAPMTs in a 128 frame circular buffer, with each frame corresponding to a GTU of $2.5 \mu \mathrm{s}$. The trigger logic on the PDM-board is thus continuously active on blocks of 320 $\mu s$. The PDM trigger logic reduces background by a factor $10^{3}$. Each PDM has a valid trigger at $7 \mathrm{~Hz}$ rate. In case of a trigger, data from a sub-group of 


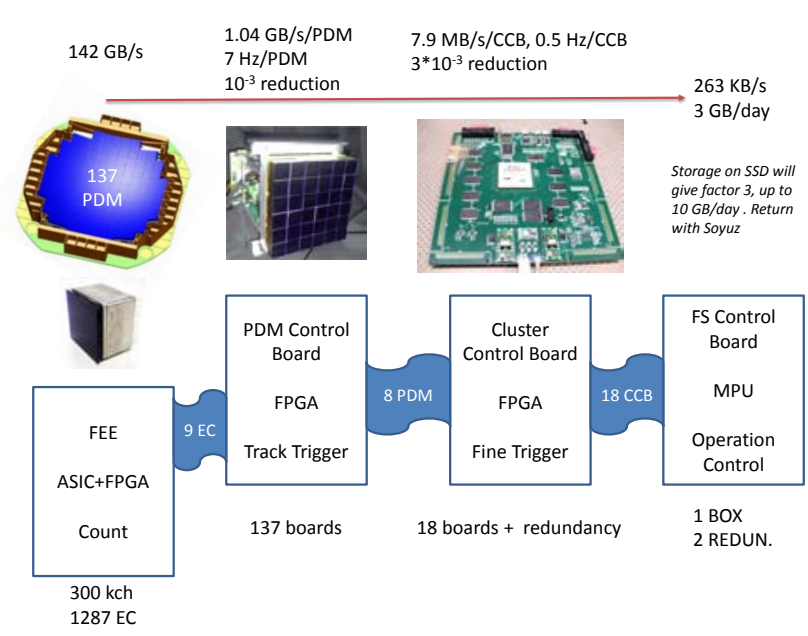

Fig. 12 Data reduction scheme. Each of the $\simeq 5000$ MAPMTs of the focal surface is read by an ASIC digitizing the photoelectron signal. A $6 \times 6$ array of MAPMT is present and read by each of the 137 PDM modules, where an FPGA performs first level triggering and rejects noise by three orders of magnitude. 8 PDMs are read by a Cluster Control Board, each with a high performance DSP which rejects noise by other three orders of magnitude. The general acquisition and data storage is performed by the main CPU.

8 PDMs are all sent to one of 18 CCBs (Cluster Control Boards) for further processing.

The CCB has the task of performing second-level-trigger, selecting potential events at a rate of $0.1 \mathrm{~Hz}$ on the whole FS. In case of a positive trigger the acquisition of the whole FS is stopped and data are sent to the CPU for storage. The whole data reduction block scheme is shown in fig. 12 . The $142 \mathrm{~GB} / \mathrm{s}$ input rate for the focal surface is the result of 8 bit data blocks for each channel for each GTU from single photon counting and 8 bits for each 8 grouped channels for each GTU from charge over time integration: 64ch · 36MAPMTs . $137 \mathrm{PDMs} \cdot 8 \mathrm{bits} \cdot \frac{1 \mathrm{~s}}{2.5 \mu \mathrm{s}}+\frac{64 \mathrm{ch}}{8} \cdot 36 \mathrm{MAPMTs} \cdot 137 \mathrm{PDMs} \cdot 8 \mathrm{bits} \cdot \frac{1 \mathrm{~s}}{2.5 \mu \mathrm{s}} \simeq 142 \frac{\mathrm{GB}}{\mathrm{s}}$.

Hot/Cold redundancy will be implemented in all systems and in all stages of data processing with the exception of intrinsically redundant devices such as the PDM detectors.

There are currently two integrated PDM units. One will be installed on the ground detector of EUSO-TA on Telescope Array site in Utah, and the second will fly on the balloon in 2014. Current TRL is 5, increasing to 6 after the balloon flight.

\subsection{PDM Front-End Electronics (FEE) - ASIC SPACIROC}

The JEM-EUSO Front-End ASIC, named SPACIROC (Spatial Photomultiplier Array Counting and Integrating ReadOut Chip) [12-14] has been designed for the readout of 64 channels MAPMTs. Its basic purpose is to per- 


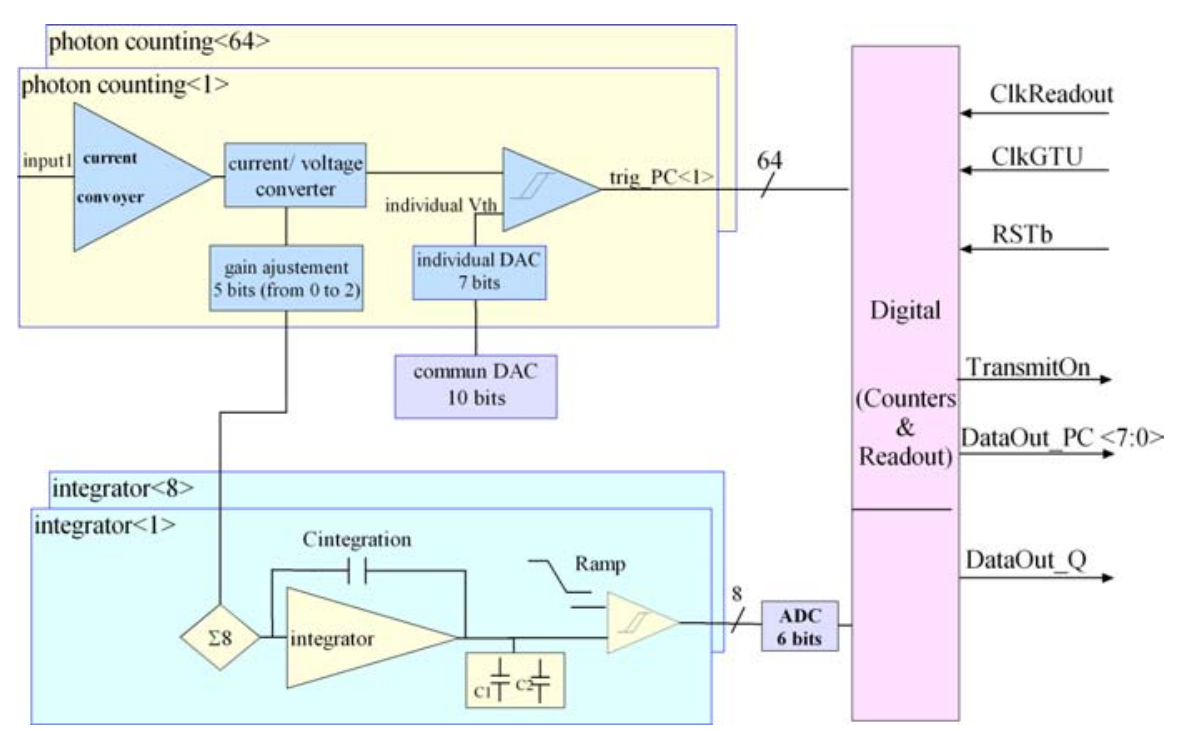

Fig. 13 SPACIROC block diagram. Top left: the analogue single photon counting part; bottom left: analogue Q-to-T readout; right: digital part.

form single photon counting from UHECR showers from a few $10^{19} \mathrm{eV}$ to $10^{21} \mathrm{eV}$ (dynamic range of 100 photons/GTU/pixel to take into account also the background). Additionally, in case of higher intensity light beyond single photon counting capabilities, typical of atmospheric phenomena, it is capable of charge-to-time (Q-to-T) conversion, extending the range of phenomena that can be observed by the telescope, with range of 0-200 photoelectrons/GTU/pixel [15]. The basic Scheme of the ASIC is shown on fig. 13. It has 64 inputs dedicated to each single anode of one MAPMT. In photon counting mode, the shapers and the discriminators are largely inherited from the MAROC3 chip [16]. Readout is carried out independently for each one of the 64 channels. The output of the photon counting discriminators and the KI comparators are processed by the digital part of the ASIC composed by a set of counters.

\section{Single photon counting}

The purpose of the digital section on this chip is to count the detected photons and to measure the intensity of the arriving photon flux by measuring the number of single photo-electrons (SPE) per pixel per GTU. The SPE are selected as signals above a threshold (one per pixel, fig. 14), fixed in the valley between pedestal and SPE. This part is also responsible for sending out the data on the serial links. Several critical areas of the digital part are coded with Triple Modular Redundancy in order to provide signal integrity, critical in space-borne applications. All the design for the digital part is optimised to minimize occupation area in order to have better performance such as a 


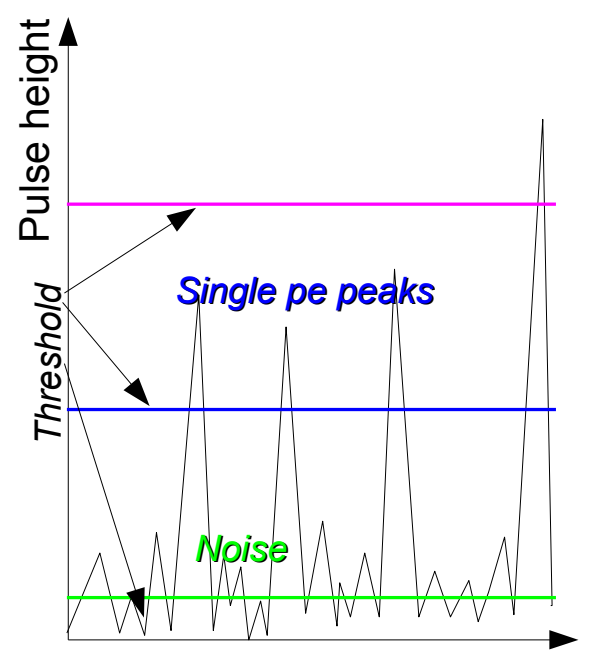

\section{Counts over threshold}

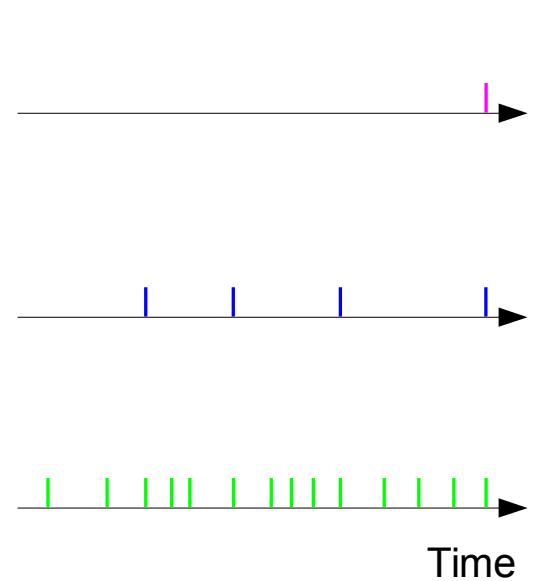

Fig. 14 A scheme of detection of single photoelectrons. Left shows a signal change with time and the right shows the counts - detection of pulses exceeding the thresholds. Small fluctuations are due to electronic noise, while big peaks is a single photoelectron signal, and the biggest peak could be a deviation of the single photoelectron or a small contribution of a double photoelectron.

reduced power consumption and robustness. Every cycle of GTU, the present data are acquired and the previously acquired data are sent out on the serial links. For the photon counting, the digital part is organised into 8 identical modules. Each module handles 8 triggers from photon counting analogue part and dumps the 64-bit data on a serial link.

The components used in the digital part are:

1. Counters: These are 8-bit Gray counters which count upon the rising edge of photon counting triggers. Every trigger from photon counting has a dedicated counter. The counters are designed for $100 \mathrm{MHz}$ clocking, thus allowing the $10 \mathrm{~ns}$ double pulses separation.

2. Counter registers: The counters data are memorised at the end of data acquisition during the current GTU. It is then sent out during the next cycle of GTU.

3. ReadOut State Machine: The state machine manages the data readout, flagging and the data checking.

The data output rate for this ASIC is 576-bit/GTU.

\section{Q-to-T readout}

In the Q-to-T converter (a.k.a KI-readout), the 64 adjusted signals are also extracted to form 8 sums of eights to feed 8 KI blocks. This converter was 
designed in collaboration with RIKEN, JAXA and Konan, Japan, with the design based on the KI02/03 chip. The input signal is passed through a lownoise and low input impedance preamplifier with an individual variable gain which allows correcting for the intrinsic non-uniformity of the MAPMT channels. Afterwards, the amplified signal is fed through shapers or into the Q-to-T converter. Currently, the chip offers 3 different triggers for each photon counting channel: one coupled with a Unipolar Fast Shaper, another with a specially designed Very Fast Shaper and the last one discriminating directly the signal coming out of the preamplifier. These come from MAROC3 technology [16] developed by Omega CNRS-IN2P3-Ecole Polytechnique microelectronics design centre.

\section{Future development}

A third generation of the ASIC, the SPACIROC-3 is under development. This shares the same cheracteristis of the previous ASIC, but has lower power consumption and cross-talk. The discriminator output will be $8 \mathrm{~ns}$ wide (instead of the current $25 \mathrm{~ns}$ ), which results in saturation above $100 \mathrm{pe} / \mathrm{GTU} /$ pixel instead of the current $30 \mathrm{pe} / \mathrm{GTU} /$ pixel. Furthermore, besides photon counting, a path is provided to charge integration information for large light events, with a coarser granularity. For this operation a sum of 8 to 16 channels is performed with individual channelwise gain adjustment in order to correct for the PMT non uniformities. The resulting sum current is integrated on a charge sensitive preamplifier to provide the charge signal per GTU over a dynamic range adjustable from 100 to 1000 pe per GTU. This charge signal is digitized on 6 bits every GTU and combined to the general readout path.

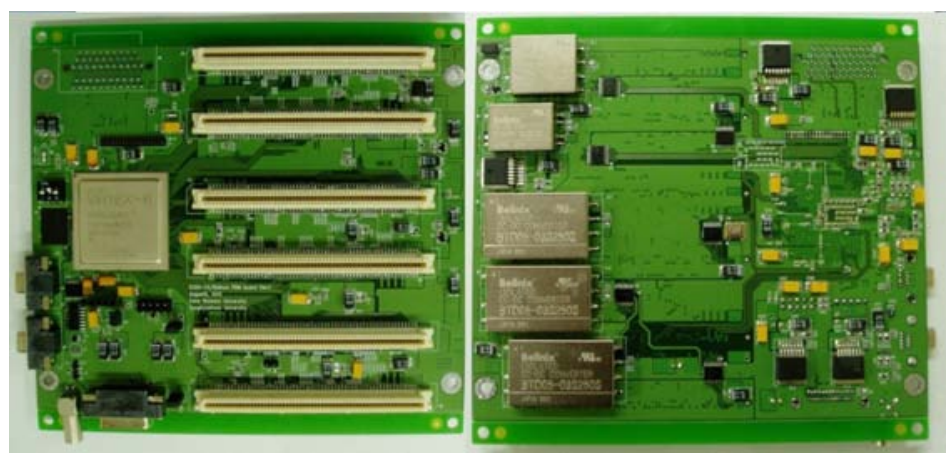

Fig. 15 PDM board, responsible for reading 36 MAPMTs through 6 EC-ASIC boards (white slots visible on the picture on the left) and performing real-time trigger. 


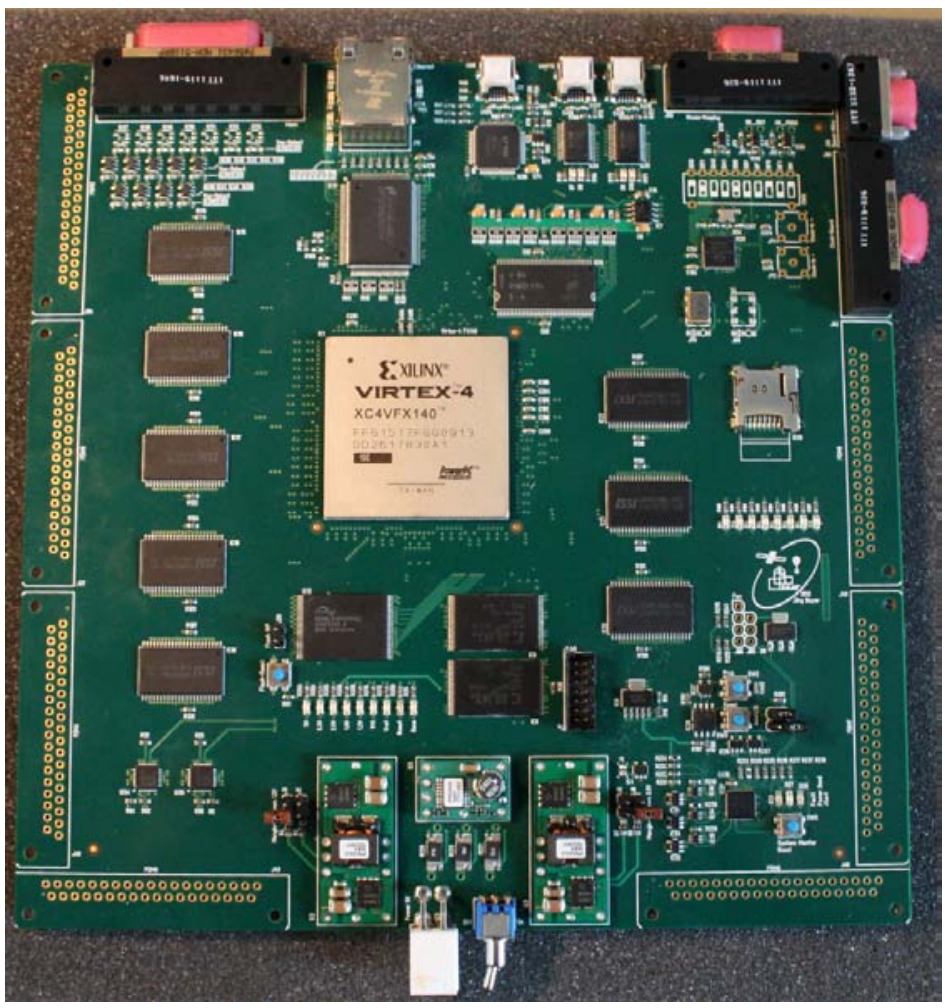

Fig. 16 The working prototype of the Cluster Control Board.

\subsection{PDM Board}

The PDM board, designed and manufactured in Korea, is devoted to read-out of signals coming from the 36 PMTs of a PDM-unit. The PDM board electronics is based on a Virtex-6 FPGA. Every $2.5 \mu$ s GTU, data (2304 channels from single photon mode $+288 \mathrm{KI}$ data) from ECs (see fig. 12) are sent to the PDM board (see fig. 15) and stored in a 128 GTU circular memory buffer. On this data the first-level trigger algorithm is continuously applied, looking for signal persistency that may be due to UV showers. Since the size of a typical event is $324 \mathrm{kB}$, full transmission is not possible, requiring $0.94 \mathrm{~GB} / \mathrm{s}$ each PDM. Therefore, as already mentioned, the task of the first level trigger is to reject the noise by a factor $10^{3}$ and pass candidate events at a maximum rate of $7 \mathrm{~Hz} / \mathrm{PDM}$ to the CCB for further processing.

\subsection{CCB Board}

The Cluster Control Board (CCB) (see fig. 16), part of the Data Processing (DP), receives data from 8 PDM boards for further processing. A Virtex- 4 
FPGA FX-60 has the task to apply the second-level-trigger to the data coming from each PDM board. In this case, a noise rejection of a factor $3 \times 10^{3}$ is expected. In case of a valid trigger the acquisition is stopped and data from relevant PDMs (possibly also linked to other CCBs) is sent via SpaceWire to the on-board CPU system. Each CCB is expected to have a trigger rate of about $0.5 \mathrm{~Hz}$, for a total Focal Surface Trigger rate of $7 \mathrm{~Hz}$. The trigger algorithm implemented to search for EAS events will be the JEM-EUSO Level 2 (L2) trigger algorithm the Linear Track Trigger (LTT). The LTT searches for tracks developing along specific directions at the speed of light. In the current design, a set of 67 predefined directions is chosen to cover the entire PDM plane homogeneously without too much overlap between the directions. In order to perform the necessary calculations as fast as possible, the hardware architecture of the trigger is highly pipelined and parallelized.

\subsection{CPU, Clock board and IDAQ board}

The CPU has low processing power $(100 \mathrm{MHz})$ compared to the PDM and CCB FPGAs, since it is in charge only of the general handling of the experiment. All the the fast operations on data handling and time synchronization are performed through two boards, the clock (CLK) board (synchronization) and the IDAQ board (data handling).

The CLK board [17] is the time synchronization system of the apparatus, it generates and distributes the system clock $(40 \mathrm{MHz})$, the GTU clock $(400$ $\mathrm{kHz}$ ) and the synchronization signal to all the devices of the FS electronics. The board generates and receives all the signals needed to control the timing of data acquisition. It is connected to the GPS system of the payload and this allows it to measure the absolute arrival time of the events with a precision of few microseconds. Another important task of the CLK board is the management of trigger signals. The board receives 2 nd level triggers from CCBs and forwards to $\mathrm{CCBs}$ any triggers coming from CPU. When the board receives a 2 nd level trigger, it goes in a busy state and sends a busy signal to CCB. In this way, the CLK Board can perform live-time and dead-time measurements. The board communicates with CPU through the IDAQ board.

The IDAQ board collects the data from the (20) CCB boards and transmits them to the CPU through a PCI bus. Communication between CCB boards and IDAQ board operate with LVDS (differential signal) to minimize interference and reduce power consumption. All lines are redundant, with each line employing double connectors at each end to increase reliability of the system and resistance to vibrations and thermal stresses. Communication protocol between IDAQ and CCB and Clock board is based on the SpaceWire protocol.

The CPU is part of the Storage and Control Unit System (SCU), the evolution of a similar system used for PAMELA [14] and is composed of a number of boards devoted to different tasks:

- CPU mainboard

- Mass Memory (8 GB) 
- Housekeeping interfaces (CAN bus)

- Interfaces to ISS (1553 and Ethernet)

- Fast bus interface for event acquisition

The CPU controls the acquisition of the experiment and general optimization of the acquisition performance in terms of data budget and detector status. It is expected to function autonomously and to reconfigure the working parameters with little or no intervention from the ground. It is capable of handling alarms and contingencies in real time, minimizing possible damage to the instrument. Long term mission operation and observation planning will be implemented from the ground with specific telecommands used to overrule the specific operation parameters of the instrument. By sending immediate or time-delayed telecommands it is possible to define the various operation parameters of the instrument in terms of specific physics objectives or specific situations.

The main CPU tasks are:

- Power on/off all subsystems

- Perform periodic calibrations

- Start acquisition / start run

- Define trigger mode acquisition

- Control information from the Housekeeping module

- Take care of real time contingency planning

- Perform periodic Download / Downlink

- Handle (slow control) 1553 commands

Slow control communication from/to ground is based on the MIL-STD1553B standard. A slow speed $(1 \mathrm{Mb} / \mathrm{s})$ bus used in space and aeronautics for transmission / reception of critical information. In JEM-EUSO the 1553 bus is employed to:

- Switch on/off the instrument or part of its sections

- Issue telecommands from the ground

- Set general acquisition parameters based on detector status

Furthermore they can be used to:

- Patch (reprogram) part of the software at CPU, DSP or FPGA levels and dump the memory of each level in case of debugging

- Receive keep-alive information from the detector, of nominal events, alarms

- Switch from main to spare channel (acquisition, power supply, etc.)

\subsection{Housekeeping and command module}

The housekeeping module - connected to the CPU - has to distribute commands to the various detectors and to collect telemetry to monitor in real-time the status of the experiment and optimize its observational parameters. HK module is capable of handling both single (upon request) or cyclic (periodic) 
acquisitions/commands. Operating both is possible according to the acquisition program and status. Different acquisitions and controls are foreseen. For instance all relays to switch on / off secondary power supplies and subsystems are controlled by high level signals. This approach has the advantage of a great degree of flexibility keeping at the same time a strong robustness and reliability.

\subsection{Storage, downlink, download}

Data stored in the mass memory of JEM-EUSO are periodically sent to ISS via a high speed link based on Ethernet protocol. Data are subsequently downlinked to the ground via Tracking and Data Relay Satellite (TDRS) link or stored on hard disks. Data transmitted to the ground consists of:

- Cosmic ray data from the focal surface

- TLEs, lightning etc.

- Housekeeping information

- Alarm

- Calibration data

- Ancillary information

Data are sent to the ground with highest priority given to housekeeping and alarm information. Experimental data are sent to ground with main priority to high energy particle data and special trigger (e.g. TLE, meteoroids, lightning, etc.). The amount of data downlinked to the Earth is $\simeq 3 \mathrm{~GB} /$ day, amounting to about $20 \%$ of the data budget. The rest of the data is stored on board ISS on a dedicated disk server. Disks are then periodically returned to the ground with Soyuz capsules or the Dragon re-entry module. In the current configuration, it is expected to have $\simeq 5$ TByte $/ 6$ months sent to the ground. Even though the UHECR event rate is very low, the background occupies a large part of the data. This is especially true at low energies, where shower development is shorter and more difficult to characterize with on board algorithms. A higher memory capability allows to increase the trigger efficiency at low energies (around) $3-4 \cdot 10^{19} \mathrm{eV}$ and improve the data bandwidth devoted to atmospheric physics (IR and UV channels).

\subsection{Atmospheric monitoring system}

The Atmospheric Monitoring system (AM) consists of an infrared camera and a laser with a steering system. Its purpose is to detect cloud height and distribution in order to correct the UV signal. The AM is described in detail in [18]. 


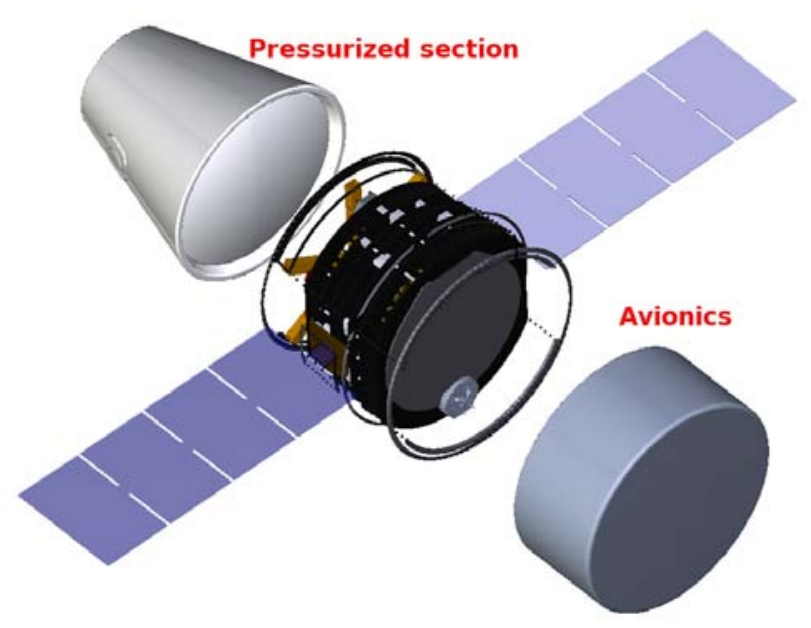

Fig. 17 3D rendering of JEM-EUSO accommodated in the Dragon launcher

\section{Mechanics}

Under the current mission profile, JEM-EUSO will be put on the ISS by Space$\mathrm{X}$ Dragon launcher ${ }^{2}$. This has required a redesign of the instrument (fig. 17), resulting in a consierable reduction of weight keeping unchanged the instrument performance and thus the science objectives. Indeed, Dragon launcher has a circular fairing requiring no side cuts; furthermore the attachment point is on the top of the rocket (toward the pressurized compartment) and not on the bottom, as in the case of HTV. This allows to have a design where the first (nadirmost) lens and support structure is much lighter, not requiring it to withstand the weight and vibrations of the launch ${ }^{3}$

The telescope is launched in a folded configuration of $177.8 \mathrm{~cm}$ heigh. After docking with the Space Station, the robotic arm pulls the instrument through the open bottom and attaches to the Kibo module. JEM-EUSO then opens through an extension mechanism to reach the needed focal length $(230 \mathrm{~cm})$ for a total height of $376.77 \mathrm{~cm}$. The telescope cylinder is nominally required to expand once for the installation. It will shrink again at the end of operational life for deorbit and disposal via Dragon (fig. 18).

A lid mechanism opens and closes the entrance pupil as the station passes from darkness to light condition and vice-versa. In case of failure of the lid mechanism the High Voltage of the PMTs can be lowered to avoid damage to the sensors in day conditions.

The telescope cylinder is divided into three lens rings (fig. 4) are extended - after coupling with the ISS - by masts driven by screws to move apart the

\footnotetext{
${ }^{2}$ Until the end of 2013 the HTV spacecraft was considered the main vehicle to transport JEM-EUSO to the orbit.

3 The HTV accommodation and design is still valid in case this launcher is used.
} 


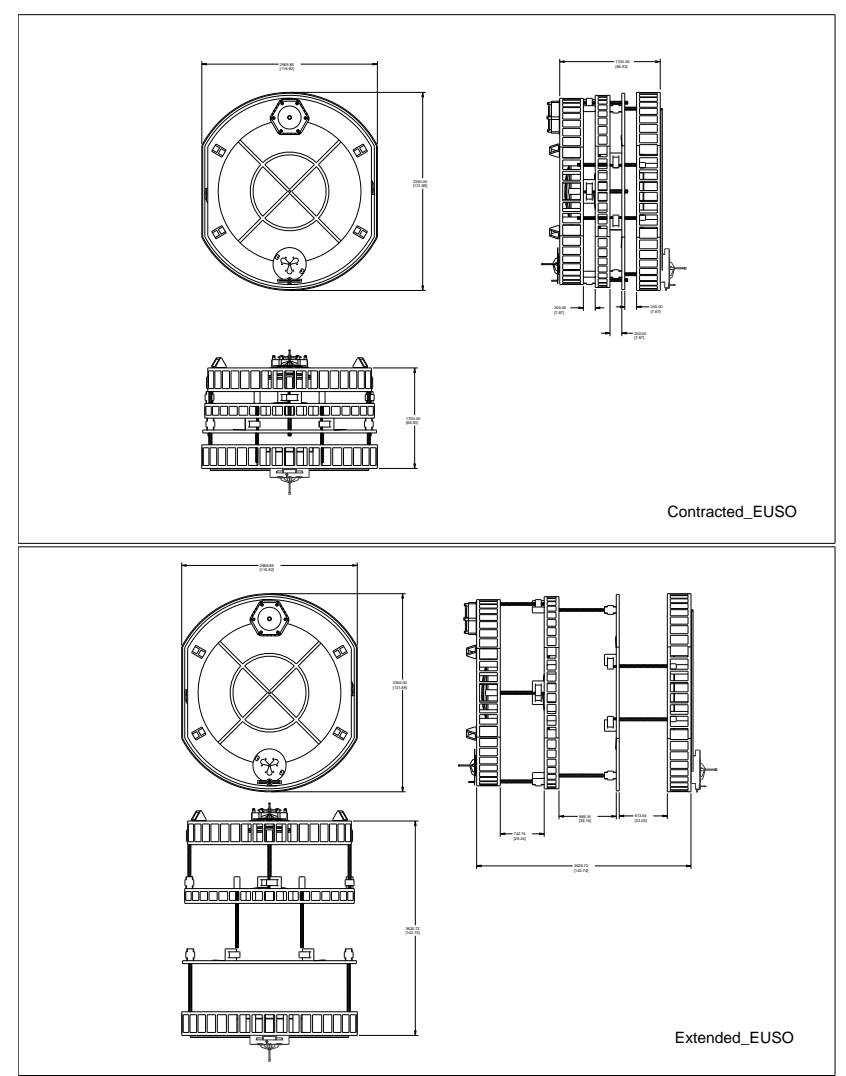

Fig. 18 JEM-EUSO dimension in the compressed (launch, top) and extended (observation, bottom) configuration.

lenses. The strength and stiffness of screws provides strength and rigidity of the structure. There are three groups of four screws each, with each group extending synchronously. This setup gives also the possibility to tilt each lens plane independently.

\section{$5.1 \mathrm{Lid}$}

The lid closes the telescope in front of the first lens, protecting the photomultipliers from light during the part of the orbit where the ISS is in local day. Thus, the lid must open and closed every orbit ( $\simeq 90$ mins). For a five year mission, this requires about 30,000 open/close iterations. In addition, it will be necessary to close the lid to protect the telescope from bad environmental conditions which are caused by approaching of HTV, re-boosting etc.

Conceptual study and design of the lid were done by the aerospace company Astro Research Co.. They confirmed the durability for much more than 30,000 
cycles of opening/closing over a 5-year period. Mass is estimated to be $20 \mathrm{~kg}$ (lid, driving mechanism, support structure, etc.). Driving power is $\simeq 3.4 \mathrm{~W}$ during the 6 seconds opening/closing operation. In the open configuration, the lid will face the velocity vector of the ISS to contribute to protecting the lenses from microdebris and atomic oxygen.

In case of failure of the lid mechanism, a backup system will leave it open. In the case that the lid in opening mode accidentally remains open even during the daytime, the focal surface may be exposed to diffusive light in the Earth daytime. We have tested that FS detector remains durable for a few years to continue the observation in the Earth night-time, as long as the high voltage is not applied. Protection to the photomultipliers will be ensured by lowering the High Voltage during local day.

\subsection{Tilt Mechanism}

The Tilt Mechanism is installed near the Payload Interface Unit (PIU) in order to tilt the whole telescope. Mission profile requires observations to begin in nadir mode, more suited to the lower energies and with a lower minimum energy threshold. After $\simeq$ one year, once spectra at low energy are obtained and matched with ground arrays, it will be possible to tilt the telescope to increase the observation area. A tilt angle of up to $30^{\circ}$ will allow the observation area to increase by about a factor of 2 . The angle of tilting can be set lower. Furthermore the tilt can be changed several times during the mission. At the end of mission tilt will return to zero to allow disposing of the instrument.

\subsection{Focal surface mechanics}

The Focal Surface is a portion of sphere of radius $2505 \mathrm{~mm}$, inserted within an in-plane section $2650 \mathrm{~mm}$ x $1900 \mathrm{~mm}$ (fig. 6). Computer Aided Design (CATIA v.5) has been used to study the FS geometry and to optimize the allocation of the various Photo-Detector Modules (PMDs) in order to maximize their number within the allocated space. The adopted configuration consists of a total of 137 PDMs arranged in a circular geometry 11 rows along the parallels of the mentioned sphere, with one PDM located at the center of FS geometry.

\section{Summary}

We have described JEM-EUSO, an instrument that will perform dedicated observations of ultra high energy cosmic rays in orbit. The observations in orbit will provide a very wide field of view and a large increase in number of observed events above existing experiments. JEM- EUSO is a UV telescope consisting of 2 Fresnel lenses and 1 Fresnel-diffractive lens, with a diameter of $2.5 \mathrm{~m}$ giving a focal length of $F \#=1$. The detection of light coming from atmospheric showers induced by UHECR is performed by a curved focal surface made 
of 4932 Multi Anode Photomultipliers, providing more than 300000 readout channels.

The JEM-EUSO telescope will be attached to the Japanese Experimental Module on board of the International Space Station. It will orbit the Earth at an altitude of $330-400 \mathrm{~km}$, every $\simeq 90$ minutes, making observations in the dark part of the Earth, about 40 minutes per orbit. Current design assumes transportation of the instrument to the ISS with a Dragon capsule.

The nadir observation mode of JEM-EUSO gives an instantaneous geometrical area greater than $1.4 \cdot 10^{5} \mathrm{~km}^{2}$, which can be increased after tilting the instrument up to $30^{\circ}$. This gives an unprecedented opportunity for observing a vast number of cosmic ray events of the highest energies, but also makes the JEM-EUSO an important experiment for general Earth observation in the UV-band, the study of atmospheric phenomena, such as Transient Luminous Events, meteors or searching for nuclearites, high energy neutrinos and heavy dark matter candidates.

Acknowledgements This paper is dedicated to the memory of Prof. Y. Takahashi.

This work was partially supported by Basic Science Interdisciplinary Research Projects of RIKEN and JSPS KAKENHI Grant (22340063, 23340081, and 24244042), by the Italian Ministry of Foreign Affairs, General Direction for the Cultural Promotion and Cooperation, by the Helmholtz Alliance for Astroparticle Physics HAP funded by the Initiative and Networking Fund of the Helmholtz Association, Germany, and by Slovak Academy of Sciences MVTS JEM-EUSO as well as VEGA grant agency project 2/0081/10. The Spanish Consortium involved in the JEM-EUSO Space Mission is funded by MICINN under projects AYA2009- 06037-E/ESP, AYA-ESP 2010-19082, AYA2011-29489-C03-01, AYA2012-39115C03-01, CSD2009-00064 (Consolider MULTIDARK) and by Comunidad de Madrid (CAM) under project S2009/ESP-1496. The work was also supported by ESA under the Topical Team program on "EUSO" by the DLR, the national aeronautics and space research centre of the Federal Republic of Germany, by Creative Research Initiatives of MEST/NRF (RCMST) in Korea and by the Mexican PAPIIT-UNAM, RedCyTE, RedFAE, CONACyT and AEM. We acknowledge the support from MAE - Italian Ministry of Foreign Affairs and INFN - National Institute of Nuclear Physics. LWP acknowledges support by JSPS.

\section{References}

1. F. Kajino, "The JEM-EUSO mission to explore the extreme universe," Nuclear Instruments and Methods in Physics Research Section A: Accelerators, Spectrometers, Detectors and Associated Equipment, vol. 623, no. 1, pp. 422 - 424, 2010. 1st International Conference on Technology and Instrumentation in Particle Physics.

2. JEM-EUSO homepage, "http://jem-euso.riken.jp."

3. J. Blecki, G. Garipov, P. Klimov, and JEM-EUSO Collaboration, "Science of atmospheric phenomena with JEM-EUSO," Experimental Astronomy, 2014.

4. A. Cellino and JEM-EUSO Collaboration, "Meteorites with JEM-EUSO," Experimental Astronomy, 2014.

5. G. Medina-Tanco, L. Anchordoqui, D. Supanitsky, and JEM-EUSO Collaboration, "Ultra High Energy Photons and Neutrinos with JEM-EUSO," Experimental Astronomy, 2014.

6. G. Medina-Tanco, K. Asano, D. Cline, T. Ebisuzaki, S. Inoue, P. Lipari, E. Parizot, A. Santangelo, G. Sigl, Y. Takahashi, H. Takami, M. Teshima, T. J. Weiler, and for the JEM-EUSO Collaboration, "JEM-EUSO Science Objectives," ArXiv e-prints, 0909.3766, Sept. 2009. 
7. Y. Takahashi and the JEM-EUSO Collaboration, "The JEM-EUSO mission," New Journal of Physics, vol. 11, pp. 065009-+, June 2009.

8. T. Ebisuzaki, Y. Uehara, H. Ohmori, K. Kawai, Y. Kawasaki, M. Sato, Y. Takizawa, M. Bertaina, F. Kajino, T. Sawabe, K. Inoue, A. Sasaki, M. Sakata, Y. Yamamoto, M. Nagano, N. Inoue, T. Shibata, N. Sakaki, Y. Uchihori, Y. Takahashi, H. Shimizu, Y. Arai, Y. Kurihara, H. Fujimoto, S. Yoshida, Y. Mizumoto, S. Inoue, K. Asano, T. Sugiyama, J. Watanabe, H. Ikeda, M. Suzuki, T. Imamura, H. Yano, T. Murakami, D. Yonetoku, Y. Itow, M. Taguchi, M. Nagata, S. Nagataki, S. Abe, T. Tajima, J. Adams, S. Mitchell, M. Christl, J. W. Jr., A. English, Y. Takahashi, K. Pitalo, J. Hadaway, J. Geary, P. Readon, H. Crawford, C. Pennypacker, K. Arisaka, D. Cline, P. Gorodetsky, P. Salin, T. Patzark, A. Maurissen, and M. Valentin, "The jem-euso project: Observing extremely high energy cosmic rays and neutrinos from the international space station," Nuclear Physics B - Proceedings Supplements, vol. 175-176, pp. $237-240,2008$. Proceedings of the XIV International Symposium on Very High Energy Cosmic Ray Interactions.

9. J. H. Adams, S. Ahmad, J.-N. Albert, D. Allard, M. Ambrosio, L. Anchordoqui, A. Anzalone, Y. Arai, C. Aramo, K. Asano, and et al., "An evaluation of the exposure in nadir observation of the JEM-EUSO mission," Astroparticle Physics, vol. 44, pp. 76-90, Apr. 2013.

10. "Amorphous fluoropolymer cytop." http://www.agc.com/english/chemicals/shinsei/cytop

11. M. Casolino, "The data acquisition and handling system of jem-euso experiment," $\mathrm{Nu}$ clear Instruments and Methods in Physics Research Section A: Accelerators, Spectrometers, Detectors and Associated Equipment, vol. 623, no. 1, pp. 516 - 518, 2010. 1st International Conference on Technology and Instrumentation in Particle Physics.

12. S. Ahmad, P. Barrillon, S. Blin-Bondil, S. Dagoret-Campagne, C. de La Taille, F. Dulucq, Y. Kawasaki, and I. Hirokazu, "Spaciroc: a rad-hard front-end readout chip for the jem-euso telescope," Journal of Instrumentation, vol. 5, no. 12, p. C12012, 2010.

13. S. Ahmad, P. Barrillon, S. Blin-Bondil, S. Dagoret-Campagne, C. D. Taille, F. Dulucq, G. Martin-Chassard, Y. Kawasaki, H. Miyamoto, H. Ikeda, T. Iguchi, and F. Kajino, "Spaciroc: A front-end readout $\{$ ASIC $\}$ for jem-euso cosmic ray observatory," Physics Procedia, vol. 37, no. 0, pp. 1600 - 1607, 2012. Proceedings of the 2nd International Conference on Technology and Instrumentation in Particle Physics (TIPP 2011).

14. S. Ahmad, P. Barrillon, S. Blin-Bondil, S. Dagoret-Campagne, C. De la Taille, F. Dulucq, G. Martin-Chassard, Y. Kawasaki, H. Miyamoto, H. Ikeda, T. Iguchi, and F. Kajino, "Spaciroc: A front-end readout asic for the jem-euso observatory," in Nuclear Science Symposium and Medical Imaging Conference (NSS/MIC), 2011 IEEE, pp. 699703,2011

15. S. Ahmad, Développement et réalisation d'un circuit de microélectronique pour le détecteur spatial de rayons cosmiques JEM-EUSO. PhD thesis, 2012.

16. S. Blin, P. Barrillon, and C. de La Taille, "Maroc, a generic photomultiplier readout chip," Journal of Instrumentation, vol. 5, no. 12, p. C12007, 2010.

17. V. Scotti, G. Osteria, and JEM-EUSO Collaboration, "The JEM-EUSO time synchronization system," Nuclear Instruments and Methods in Physics Research A, vol. 718, pp. 248-250, Aug. 2013.

18. J.-E. collaboration, "The atmospheric monitoring system of the jem-euso instrument," this journal.

\section{The JEM-EUSO Collaboration}

J.H. Adams Jr. ${ }^{m d}$, S. Ahmad ${ }^{b b}$, J.-N. Albert ${ }^{b a}$, D. Allard ${ }^{b c}$, L. Anchordoqui ${ }^{m f}$, V. Andreev ${ }^{m e}$,

A. Anzalone ${ }^{d h, d n}$, Y. Arai ${ }^{e v}$, K. Asano ${ }^{e t}$, M. Ave Pernas ${ }^{k c}$, P. Baragatti ${ }^{d o}$, P. Barrillon ${ }^{b a}$,

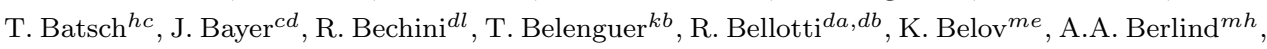

M. Bertaina ${ }^{d k, d l}$, P.L. Biermann ${ }^{c b}$, S. Biktemerova ${ }^{i a}$, C. Blaksley ${ }^{b c}$, N. Blanc ${ }^{l a}$, J. Błęcki $^{h d}$,

S. Blin-Bondil ${ }^{b b}$, J. Blümer ${ }^{c b}$, P. Bobik ${ }^{j a}$, M. Bogomilov $^{a a}$, M. Bonamente ${ }^{m d}$, M.S. Briggs ${ }^{m d}$,

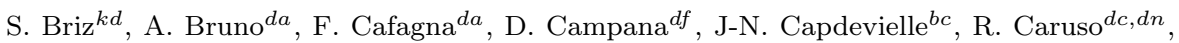

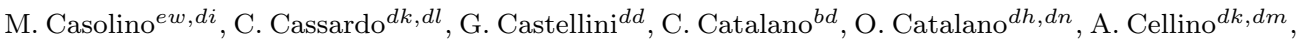


M. Chikawa ${ }^{e d}$, M.J. Christl ${ }^{m g}$, D. Cline ${ }^{m e}$, V. Connaughton ${ }^{m d}$, L. Conti ${ }^{d o}$, G. Cordero ${ }^{g a}$, H.J. Crawford ${ }^{m a}$, R. Cremonini ${ }^{d l}$, S. Csorna ${ }^{m h}$, S. Dagoret-Campagne ${ }^{b a}$, A.J. de Castro ${ }^{k d}$, C. De Donato ${ }^{d i}$, C. de la Taille ${ }^{b b}$, C. De Santis ${ }^{d i, d j}$, L. del Peral ${ }^{k c}$, A. Dell'Oro ${ }^{d k, d m}$ N. De Simone $^{d i}$, M. Di Martino ${ }^{d k, d m}$, G. Distratis ${ }^{c d}$, F. Dulucq ${ }^{b b}$, M. Dupieux $^{b d}$, A. Ebersoldt ${ }^{c b}$,

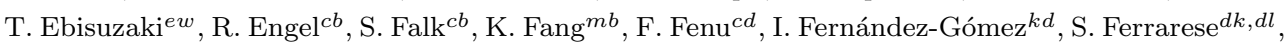
D. Finco ${ }^{d o}$, M. Flamini ${ }^{d o}$, C. Fornaro ${ }^{d o}$, A. Franceschi ${ }^{d e}$, J. Fujimoto ${ }^{e v}$, M. Fukushima ${ }^{e g}$, P. Galeotti ${ }^{d k, d l}$, G. Garipov ${ }^{i c}$, J. Geary ${ }^{m d}$, G. Gelmini ${ }^{m e}$, G. Giraudo ${ }^{d k}$, M. Gonchar ${ }^{i a}$, C. González Alvarado ${ }^{k b}$, P. Gorodetzky ${ }^{b c}$, F. Guarino ${ }^{d f, d g}$, A. Guzmán ${ }^{c d}$, Y. Hachisu ${ }^{e w}$, B. Harlov ${ }^{i b}$, A. Haungs ${ }^{c b}$, J. Hernández Carretero ${ }^{k c}$, K. Higashide $^{e r, e w}$, D. Ikeda ${ }^{e g}$, H. Ikeda $^{e p}$,

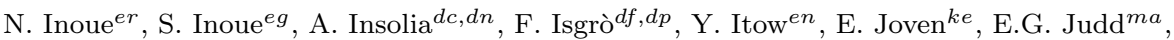
A. Jung ${ }^{f b}$, F. Kajino ${ }^{e i}$, T. Kajino ${ }^{e l}$, I. Kaneko ${ }^{e w}$, Y. Karadzhov ${ }^{a a}$, J. Karczmarczyk ${ }^{h c}$, M. Karus ${ }^{c b}$, K. Katahira ${ }^{e w}$, K. Kawai ${ }^{e w}$, Y. Kawasaki ${ }^{e w}$, B. Keilhauer ${ }^{c b}$, B.A. Khrenov ${ }^{i c}$, Jeong-Sook Kim ${ }^{f a}$, Soon-Wook Kim ${ }^{f a}$, Sug-Whan Kim ${ }^{f d}$, M. Kleifges ${ }^{c b}$, P.A. Klimov ${ }^{i c}$, D. Kolev ${ }^{a a}$, I. Kreykenbohm ${ }^{c a}$, K. Kudela ${ }^{j a}$, Y. Kurihara ${ }^{e v}$, A. Kusenko ${ }^{m e}$, E. Kuznetsov ${ }^{m d}$,

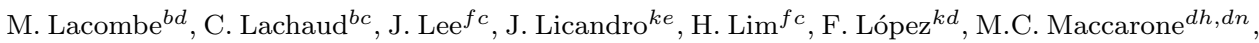

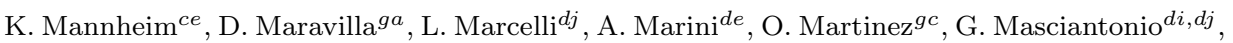
K. Mase ${ }^{e a}$, R. Matev ${ }^{a a}$, G. Medina-Tanco ${ }^{g a}$, T. Mernik ${ }^{c d}$, H. Miyamoto ${ }^{b a}$, Y. Miyazaki $^{e c}$,

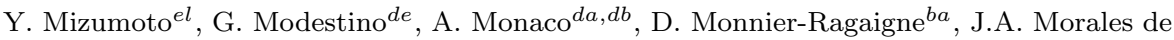
los Ríos ${ }^{k a, k c}$, C. Moretto ${ }^{b a}$, V.S. Morozenko ${ }^{i c}$, B. Mot $^{b d}$, T. Murakami ${ }^{e f}$, M. Nagano ${ }^{e c}$, M. Nagata ${ }^{e h}$, S. Nagataki ${ }^{e k}$, T. Nakamura ${ }^{e j}$, T. Napolitano ${ }^{d e}$, D. Naumov ${ }^{i a}$, R. Nava ${ }^{g a}$, A. Neronov ${ }^{l b}$, K. Nomoto ${ }^{e u}$, T. Nonaka ${ }^{e g}$, T. Ogawa ${ }^{e w}$, S. Ogio ${ }^{e o}$, H. Ohmori ${ }^{e w}$, A.V. Olinto ${ }^{m b}$, P. Orleański ${ }^{h d}$, G. Osteria ${ }^{d f}$, M.I. Panasyuk ${ }^{i c}$, E. Parizot ${ }^{b c}$, I.H. Park ${ }^{f c}$, H.W. Park ${ }^{f c}$, B. Pastircak ${ }^{j a}$, T. Patzak $^{b c}$, T. Paul ${ }^{m f}$, C. Pennypacker ${ }^{m a}$, S. Perez Cano ${ }^{k c}$, T. Peter ${ }^{l c}$, P. Picozza ${ }^{d i, d j, e w}$, T. Pierog ${ }^{c b}$, L.W. Piotrowski ${ }^{e w}$, S. Piraino ${ }^{c d, d h}$, Z. Plebaniak ${ }^{h c}$, A. Pollini ${ }^{l a}$,

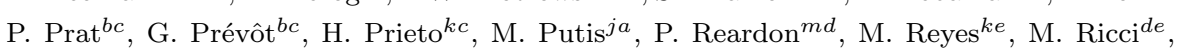
I. Rodríguez ${ }^{k d}$, M.D. Rodríguez Frías ${ }^{k c}$, F. Ronga ${ }^{d e}$, M. Roth $^{c b}$, H. Rothkaehl ${ }^{h d}$, G. Roudil ${ }^{b d}$, I. Rusinov ${ }^{a a}$, M. Rybczyński ${ }^{h a}$, M.D. Sabau ${ }^{k b}$, G. Sáez Cano ${ }^{k c}$, H. Sagawa ${ }^{e g}$, A. Saito ${ }^{e j}$, N. Sakaki ${ }^{c b}$, M. Sakata ${ }^{e i}$, H. Salazar ${ }^{g c}$, S. Sánchez ${ }^{k d}$, A. Santangelo ${ }^{c d}$, L. Santiago Crúz ${ }^{g a}$, M. Sanz Palomino ${ }^{k b}$, O. Saprykin ${ }^{i b}$, F. Sarazin ${ }^{m c}$, H. Sato ${ }^{e i}$, M. Sato ${ }^{e s}$, T. Schanz ${ }^{c d}$, H. Schieler ${ }^{c b}$, V. Scotti ${ }^{d f, d g}$, A. Segreto ${ }^{d h, d n}$, S. Selmane ${ }^{b c}$, D. Semikoz ${ }^{b c}$, M. Serra ${ }^{k e}$, S. Sharakin ${ }^{i c}$, T. Shibata ${ }^{e q}$, H.M. Shimizu ${ }^{e m}$, K. Shinozaki ${ }^{e w}, c d$, T. Shirahama ${ }^{e r}$, G. SiemieniecOziębło $^{h b}$, H.H. Silva López ${ }^{g a}$, J. Sledd ${ }^{m g}$, K. Słomińska ${ }^{h d}$, A. Sobey ${ }^{m g}$, T. Sugiyama ${ }^{e m}$, D. Supanitsky ${ }^{g a}$, M. Suzuki ${ }^{e p}$, B. Szabelska ${ }^{h c}$, J. Szabelski $^{h c}$, F. Tajima ${ }^{e e}$, N. Tajima ${ }^{e w}$, T. Tajima ${ }^{c c}$, Y. Takahashi ${ }^{e s}$, H. Takami ${ }^{e v}$, M. Takeda ${ }^{e g}$, Y. Takizawa ${ }^{e w}$, C. Tenzer ${ }^{c d}$,

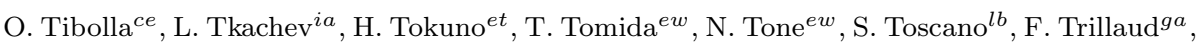
R. Tsenov ${ }^{a a}$, Y. Tsunesada ${ }^{e t}, \mathrm{~K}$. Tsuno ${ }^{e w}$, T. Tymieniecka ${ }^{h c}$, Y. Uchihori ${ }^{e b}$, M. Unger $^{c b}$, O. Vaduvescu $^{k e}$, J.F. Valdés-Galicia ${ }^{g a}$, P. Vallania ${ }^{d k, d m}$, L. Valore ${ }^{d f, d g}$, G. Vankova ${ }^{a a}$, C. Vigorito ${ }^{d k, d l}$, L. Villaseñor ${ }^{g b}$, P. von Ballmoos ${ }^{b d}, \mathrm{~S}_{\text {. Wada }}{ }^{e w}, \mathrm{~J}$. Watanabe ${ }^{e l}, \mathrm{~S}$. Watanabe ${ }^{e s}$, J. Watts Jr. ${ }^{m d}$, M. Weber ${ }^{c b}$, T.J. Weiler ${ }^{m h}$, T. Wibig ${ }^{h c}$, L. Wiencke ${ }^{m c}$, M. Wille ${ }^{c a}$, J. Wilms $^{c a}$,

Z. Włodarczyk ${ }^{h a}$, T. Yamamoto ${ }^{e i}$, Y. Yamamoto ${ }^{e i}$, J. Yang ${ }^{f b}$, H. Yano ${ }^{e p}$, I.V. Yashin ${ }^{i c}$,

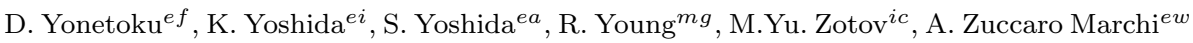

aa St. Kliment Ohridski University of Sofia, Bulgaria

${ }^{b a}$ LAL, Univ Paris-Sud, CNRS/IN2P3, Orsay, France

${ }^{b b}$ Omega, Ecole Polytechnique, CNRS/IN2P3, Palaiseau, France

${ }^{b c}$ APC, Univ Paris Diderot, CNRS/IN2P3, CEA/Irfu, Obs. de Paris, Sorbonne Paris Cité, France

${ }^{b d}$ IRAP, Université de Toulouse, CNRS, Toulouse, France 
ca ECAP, University of Erlangen-Nuremberg, Germany

${ }^{c b}$ Karlsruhe Institute of Technology (KIT), Germany

${ }^{c c}$ Ludwig Maximilian University, Munich, Germany

${ }^{c d}$ Inst. for Astronomy and Astrophysics, Kepler Center, University of Tübingen, Germany

ce Institut für Theoretische Physik und Astrophysik, University of Würzburg, Germany

da Istituto Nazionale di Fisica Nucleare - Sezione di Bari, Italy

$d b$ Universita' degli Studi di Bari Aldo Moro and INFN - Sezione di Bari, Italy

$d c$ Dipartimento di Fisica e Astronomia - Universita' di Catania, Italy

${ }^{d d}$ Consiglio Nazionale delle Ricerche (CNR) - Ist. di Fisica Applicata Nello Carrara, Firenze, Italy

de Istituto Nazionale di Fisica Nucleare - Laboratori Nazionali di Frascati, Italy

$d f$ Istituto Nazionale di Fisica Nucleare - Sezione di Napoli, Italy

$d g$ Universita' di Napoli Federico II - Dipartimento di Scienze Fisiche, Italy

$d h$ INAF - Istituto di Astrofisica Spaziale e Fisica Cosmica di Palermo, Italy

di Istituto Nazionale di Fisica Nucleare - Sezione di Roma Tor Vergata, Italy

dj Universita' di Roma Tor Vergata - Dipartimento di Fisica, Roma, Italy

$d k$ Istituto Nazionale di Fisica Nucleare - Sezione di Torino, Italy

$d l$ Dipartimento di Fisica, Universita' di Torino, Italy

$d m$ Osservatorio Astrofisico di Torino, Istituto Nazionale di Astrofisica, Italy

$d n$ Istituto Nazionale di Fisica Nucleare - Sezione di Catania, Italy

do UTIU, Dipartimento di Ingegneria, Rome, Italy

$d p$ DIETI, Universita' degli Studi di Napoli Federico II, Napoli, Italy

ea Chiba University, Chiba, Japan

${ }^{e b}$ National Institute of Radiological Sciences, Chiba, Japan

ec Fukui University of Technology, Fukui, Japan

ed Kinki University, Higashi-Osaka, Japan

ee Hiroshima University, Hiroshima, Japan

ef Kanazawa University, Kanazawa, Japan

eg Institute for Cosmic Ray Research, University of Tokyo, Kashiwa, Japan

eh Kobe University, Kobe, Japan

ei Konan University, Kobe, Japan

ej Kyoto University, Kyoto, Japan

ek Yukawa Institute, Kyoto University, Kyoto, Japan

el National Astronomical Observatory, Mitaka, Japan

em Nagoya University, Nagoya, Japan

en Solar-Terrestrial Environment Laboratory, Nagoya University, Nagoya, Japan

eo Graduate School of Science, Osaka City University, Japan

${ }^{e p}$ Institute of Space and Astronautical Science/JAXA, Sagamihara, Japan

eq Aoyama Gakuin University, Sagamihara, Japan

er Saitama University, Saitama, Japan

es Hokkaido University, Sapporo, Japan

et Interactive Research Center of Science, Tokyo Institute of Technology, Tokyo, Japan

eu University of Tokyo, Tokyo, Japan

ev High Energy Accelerator Research Organization (KEK), Tsukuba, Japan

ew RIKEN, Wako, Japan

fa Korea Astronomy and Space Science Institute (KASI), Daejeon, Republic of Korea

$f b$ Ewha Womans University, Seoul, Republic of Korea 
${ }^{f c}$ Sungkyunkwan University, Seoul, Republic of Korea

${ }^{f d}$ Center for Galaxy Evolution Research, Yonsei University, Seoul, Republic of Korea

ga Universidad Nacional Autónoma de México (UNAM), Mexico

${ }^{g b}$ Universidad Michoacana de San Nicolas de Hidalgo (UMSNH), Morelia, Mexico

${ }^{g c}$ Benemérita Universidad Autónoma de Puebla (BUAP), Mexico

ha Jan Kochanowski University, Institute of Physics, Kielce, Poland

$h b$ Jagiellonian University, Astronomical Observatory, Krakow, Poland

$h c$ National Centre for Nuclear Research, Lodz, Poland

${ }^{h d}$ Space Research Centre of the Polish Academy of Sciences (CBK), Warsaw, Poland

ia Joint Institute for Nuclear Research, Dubna, Russia

${ }^{i b}$ Central Research Institute of Machine Building, TsNIIMash, Korolev, Russia

ic Skobeltsyn Institute of Nuclear Physics, Lomonosov Moscow State University, Russia

ja Institute of Experimental Physics, Kosice, Slovakia

ka Consejo Superior de Investigaciones Científicas (CSIC), Madrid, Spain

${ }^{k b}$ Instituto Nacional de Técnica Aeroespacial (INTA), Madrid, Spain

${ }^{k c}$ Universidad de Alcalá (UAH), Madrid, Spain

${ }^{k d}$ Universidad Carlos III de Madrid, Spain

ke Instituto de Astrofísica de Canarias (IAC), Tenerife, Spain

la Swiss Center for Electronics and Microtechnology (CSEM), Neuchâtel, Switzerland

${ }^{l b}$ ISDC Data Centre for Astrophysics, Versoix, Switzerland

${ }^{l c}$ Institute for Atmospheric and Climate Science, ETH Zürich, Switzerland

$m a$ Space Science Laboratory, University of California, Berkeley, USA

$m b$ University of Chicago, USA

$m c$ Colorado School of Mines, Golden, USA

$m d$ University of Alabama in Huntsville, Huntsville, USA

me University of California (UCLA), Los Angeles, USA

$m f$ University of Wisconsin-Milwaukee, Milwaukee, USA

$m g$ NASA - Marshall Space Flight Center, USA

$m h$ Vanderbilt University, Nashville, USA 